\title{
Pharmacological Discrimination of Extinction and Reconsolidation of Contextual Fear Memory by a Potentiator of AMPA Receptors
}

\author{
Daisuke Yamada ${ }^{1,2}$, Ko Zushida ${ }^{1,2}$, Keiji Wada ${ }^{1,2}$ and Masayuki Sekiguchi*,, ${ }^{, 2}$ \\ 'Department of Degenerative Neurological Diseases, National Institute of Neuroscience, National Center of Neurology and Psychiatry, Kodaira, \\ Tokyo, Japan; ${ }^{2}$ CREST, Japan Science and Technology Agency, Kawaguchi, Saitama, Japan
}

\begin{abstract}
Conditioned fear memory, once formed through fear conditioning, is modulated by reexposure of individuals to a conditioned stimulus. The reexposure reactivates the fear memory, which induces reconsolidation of the memory first, and then extinction of the fear response. Both attenuating the former and facilitating the latter are effective in reducing the fear response, and these findings are potentially translatable to the enhancement of exposure therapy for complex anxiety disorders. Currently, there is no drug that is established to modulate either reconsolidation or extinction selectively, which are thought to be independent processes. Here, we report that an extinction-facilitating AMPA potentiator, 4-[2-(phenylsulfonylamino)ethylthio]-2,6-difluoro-phenoxyacetamide (PEPA), does not act on the reconsolidation of fear memory formed by contextual fear conditioning in mice. The freezing rates observed in contextually conditioned mice following short reexposure $(3 \mathrm{~min})$ to the context were not influenced by intraperitoneal or intra-amygdala administration of PEPA. The same short reexposure to the context enhanced freezing responses in mice that were similarly administered D-cycloserine (DCS), a drug that facilitates both extinction and reconsolidation, and this enhancement of freezing responses in mice intraperitoneally administered DCS was abolished by propranolol, a drug that suppresses reconsolidation. At the same doses used in the short reexposure experiments, PEPA and DCS facilitated extinction of the fear response induced by long reexposure to the context and suppressed reinstatement of the conditioned fear memory. PEPA and DCS did not affect reextinction. These results suggest that PEPA acts on extinction of contextual fear memory without having detectable influences on its reconsolidation.
\end{abstract}

Neuropsychopharmacology (2009) 34, 2574-2584; doi:I0.1038/npp.2009.86; published online 12 August 2009

Keywords: fear memory; reconsolidation; extinction; PEPA; D-cycloserine; exposure therapy

\section{INTRODUCTION}

Conditioned fear memory, once formed through Pavlovian fear conditioning, is modulated by reexposure of individuals to a conditioned stimulus (CS). The reexposure reactivates the fear memory, which induces reconsolidation of the memory first, and then extinction of the fear response (Myers and Davis, 2002; Ouyang and Thomas, 2005; Suzuki et al, 2004). Reconsolidation is a process that results in a persistent or greater fear response once consolidated memory is destabilized and reconstructed into a more stable state by short exposure to a CS (Sara, 2000; Nader et al, 2000). Extinction is a process by which a new inhibitory association is formed following a longer exposure to a CS, resulting in a reduced fear response (Myers and Davis, 2002). Previous studies suggest that the basolateral

*Correspondence: Dr M Sekiguchi, Department of Degenerative Neurological Diseases, National Institute of Neuroscience, National Center of Neurology and Psychiatry, 4-I-I Ogawahigashi, Kodaira, Tokyo 187-8502, Japan, Tel: +8I 42346 1715, Fax: +8I 42346 1745, E-mail: elecl@ncnp.go.jp or sekiguch@ncnp.go.jp

Received 2 February 2009; revised 2 July 2009; accepted 2 July 2009 nucleus of the amygdala (BLA) is mainly involved in reconsolidation (Nader et al, 2000; Duvarci and Nader, 2004; Baldi et al, 2008), and that the amygdala, hippocampus, and medial prefrontal cortex (mPFC) form a circuit necessary for extinction in rodents (for review, see Corcoran and Quirk, 2007; Sotres-Bayon et al, 2004; Quirk and Mueller, 2008).

Both attenuating reconsolidation and facilitating extinction are effective to reduce the emergence of a fear response (Diergaarde et al, 2008; Davis et al, 2006). From a clinical point of view, this strategy has attracted attention as a method to enhance exposure therapy for complex anxiety disorders such as phobias, post-traumatic stress disorder (PTSD), and obsessive-compulsive disorder. Based on this strategy, a partial agonist for NMDA receptors, D-cycloserine (DCS), a highly studied extinction-facilitating drug, was applied to humans in a clinical trial to enhance exposure therapy for acrophobia, social anxiety disorder, and obsessive-compulsive disorder, and was confirmed to enhance exposure therapy (Ressler et al, 2004; Hofmann et al, 2006; Kushner et al, 2007; Guastella et al, 2008; Vervliet, 2008; Norberg et al, 2008). 
Reconsolidation and extinction are thought to be independent processes. However, because common molecular mechanisms, such as protein synthesis and activation of NMDA receptors, $\beta$-adrenergic receptors, protein kinase $A$, and mitogen-activated protein kinases, are involved in these two processes (for review, see Tronson and Taylor, 2007; Myers and Davis, 2007), drugs that act on extinction may also influence reconsolidation. Therefore, at present, to manipulate either of the two processes with systemic pharmacological reagents, strict control of the duration of reexposure is required to optimize the conditions for each process (Suzuki et al, 2004). Specifically, with long reexposures systemic DCS attenuates fear responses by facilitating extinction, but with shorter reexposures it enhances fear responses by facilitating reconsolidation (Walker et al, 2002; Ledgerwood et al, 2003; Lee et al, 2006). Conversely, with short reexposures systemic propranolol (PROP) attenuates fear responses by suppressing reconsolidation, but with long reexposures it enhances fear responses by reducing extinction (Ouyang and Thomas, 2005; Debiec and LeDoux, 2004; Mueller et al, 2008). Therefore, seeking a compound that selectively acts on reconsolidation or extinction is potentially important for two reasons: (1) to provide a pharmacological demonstration that reconsolidation and extinction are independent processes; and (2) to facilitate development of a therapeutic drug that enhances exposure therapy without the undesired side effect of fear augmentation. For the second aspect, a compound that could be administered systemically would be desirable.

Previously, we reported (Zushida et al, 2007) that intraperitoneal or intra-mPFC administration of the AMPA potentiator, 4-[2-(phenylsulfonylamino)ethylthio]-2,6-difluorophenoxyacetamide (PEPA), facilitates extinction learning of contextual fear in mice, and that this facilitation is mainly due to activation of the MPFC because (1) the potentiating actions of PEPA on the excitatory synaptic response recorded in BLA pyramidal neurons and hippocampal CA1 pyramidal neurons were weaker than those on the synaptic response recorded in mPFC pyramidal neurons, and (2) intra-amygdala administration of PEPA did not significantly facilitate extinction learning. These weaker electrophysiological and behavioral actions of PEPA on the BLA prompted us to hypothesize that PEPA may not act on reconsolidation of fear memory, because the BLA is mainly involved in reconsolidation as mentioned above. In the present study, based on this hypothesis, we examined the action of PEPA on reconsolidation of fear memory in mice, and found that intraperitoneal or intra-amygdala administration of PEPA did not influence fear memory reconsolidation. Furthermore, the actions of PEPA and DCS on extinction, and another two extinction-related behaviors, reinstatement and reextinction, were also compared to characterize the properties of PEPA as an extinctionfacilitating drug.

\section{MATERIALS AND METHODS}

\section{Animals}

Male C57BL/6J mice were purchased from Clea (Tokyo, Japan) and used in experiments after habituation to the environment of our institute's animal center for at least 1 week. The mice were 10 - to 12 -week old at the beginning of experiments. The mice were housed four or five per cage under controlled conditions of temperature $\left(25 \pm 1{ }^{\circ} \mathrm{C}\right)$ and lighting ( $12 \mathrm{~h}$ light/dark cycle) and provided with food and water ad libitum. All experiments were conducted in strict accordance with the regulations of the National Institute of Neuroscience (Japan) for animal experiments.

\section{Apparatus}

All behavioral experiments were conducted in the same conditioning chamber $(20 \times 20 \mathrm{~cm}$ bottom, $35 \mathrm{~cm}$ high; Muromachi Kikai, Tokyo, Japan) inside a sound-attenuating box. The chamber was made of white polyvinyl chloride, exception for the grid floor, which was made of stainless steel bars $(0.5 \mathrm{~cm}$ in diameter, spaced $1.0 \mathrm{~cm}$ apart $)$. The rods were wired to a shock generator/scrambler (SGS-002; Muromachi Kikai) for the delivery of electrical footshocks. A digital video camera connected to a VHS video recorder was mounted above the chambers to permit video scoring of the animal's behavior. The illumination on the floor of the box was 200 lux.

\section{Drugs}

For intraperitoneal administration, PEPA (Sigma, St Louis, MO; or kindly supplied by Nihon Suisan, Tokyo, Japan) and DCS (Sigma) were dissolved ( $3 \mathrm{~g} / \mathrm{l})$ into vehicle consisting of $0.45 \% \mathrm{NaCl}$ and $33 \%$ (2-hydroxypropyl)- $\beta$-cyclodextrin by shaking vigorously more than $3 \mathrm{~h}$ (in case of PEPA). ( \pm )-Propranolol hydrochloride (Sigma) was dissolved in saline $(1 \mathrm{~g} / \mathrm{l})$. These drug solutions were administered into mice at a dose of $30 \mathrm{mg} / \mathrm{kg}$ body weight (volume $10 \mathrm{ml} / \mathrm{kg}$ ), except for PROP, which was injected at a dose of $10 \mathrm{mg} / \mathrm{kg}$. The timings of injections varied among experiments and are described in the Results section. For intra-amygdala administration, PEPA and DCS were dissolved $(2.5 \mathrm{~g} / \mathrm{l})$ into vehicle (17\% (2-hydroxypropyl)- $\beta$-cyclodextrin solution).

\section{Behavioral Procedures}

Grouping. Before each experiment, the mice used in the experiment were randomly divided into two (Figure 1a-c and $g-i$ ) or three groups (Figure 1d-f; Figures 2-5). The mice in each group were assigned to the administration of vehicle or drugs, according to the schedule below.

Experiment 1: effects of PEPA and DCS on reconsolidation of contextual fear memory. On day 1 (Conditioning), mice were placed in the conditioning chamber and permitted to explore the chamber. After $148 \mathrm{~s}$, a single electrical footshock was delivered $(0.8 \mathrm{~mA}, 2 \mathrm{~s}$ duration). Mice were removed from the chamber $30 \mathrm{~s}$ after the end of the footshock and returned to their home cages. On day 2 (Reexposure), mice were reexposed to the conditioning chamber without footshock for $3 \mathrm{~min}$, for memory reactivation. In the experiment shown in Figure 1a-c, vehicle $-15 \mathrm{~min}$ and vehicle $-30 \mathrm{~min}$ groups were intraperitoneally injected with vehicle 15 and $30 \mathrm{~min}$ before the Reexposure session. In the experiment shown in Figure $1 \mathrm{~d}-\mathrm{f}$, vehicle and PEPA groups were intraperitoneally injected with 

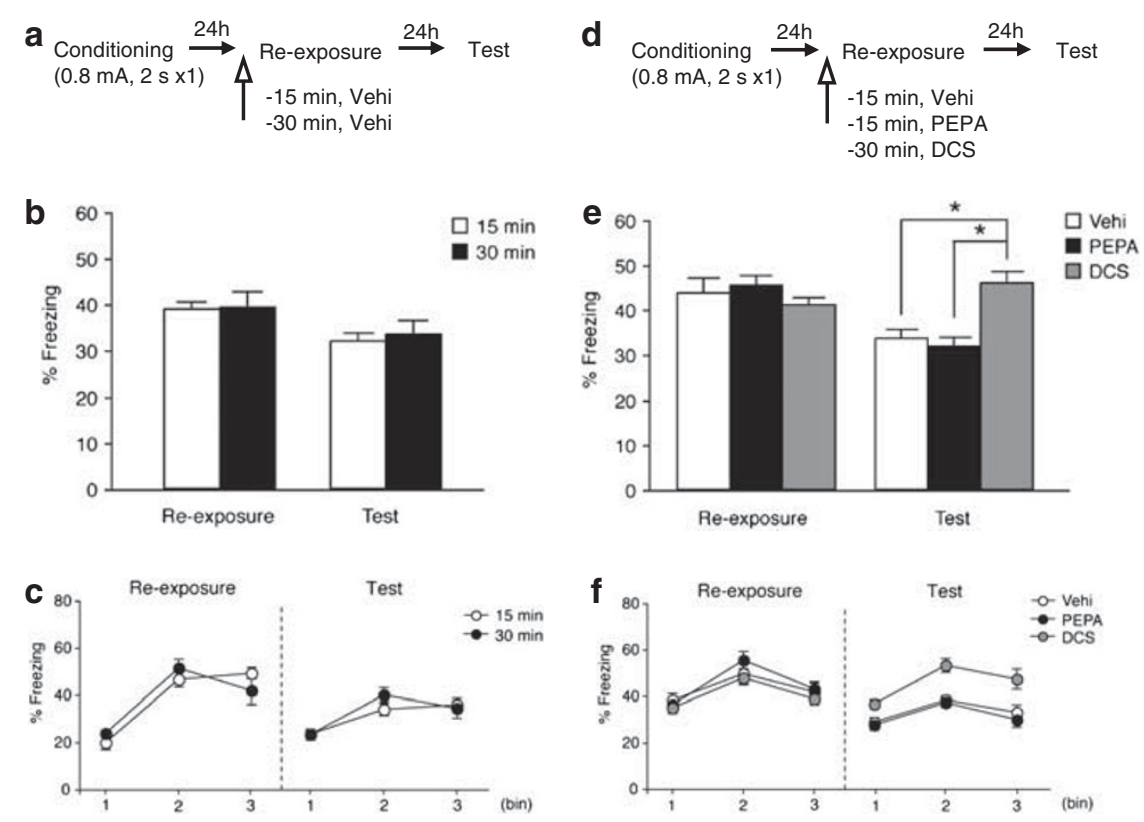

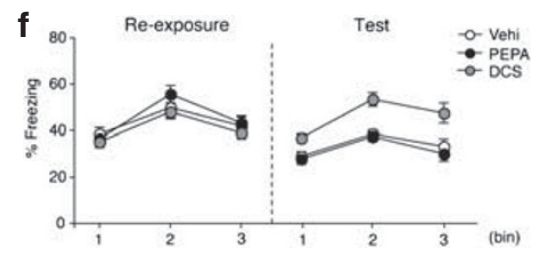

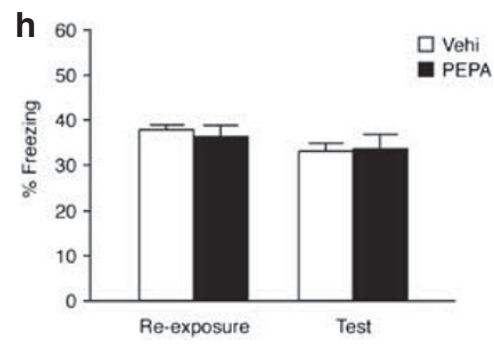
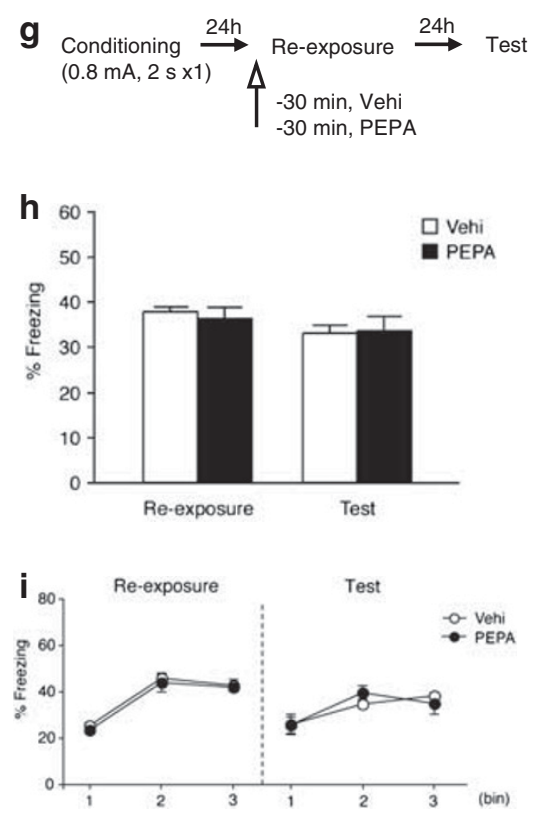

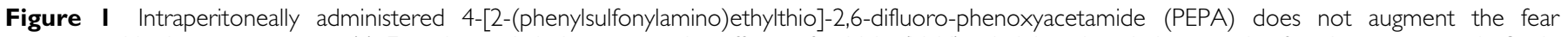

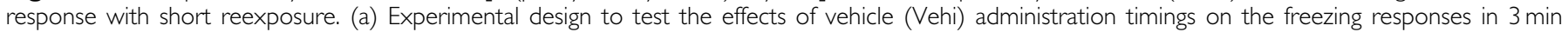

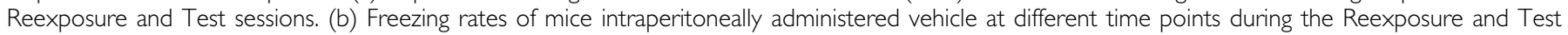

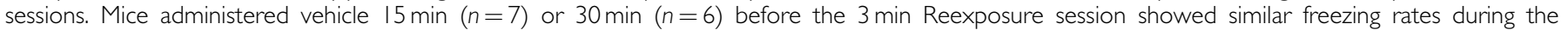

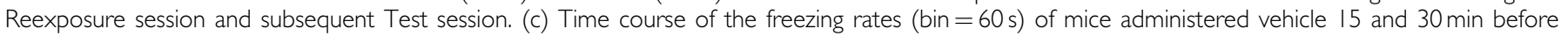

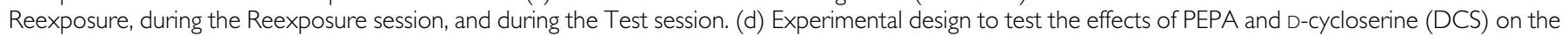

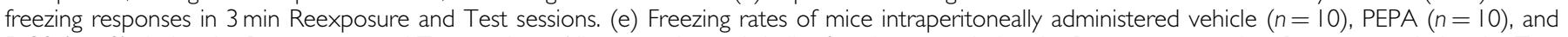

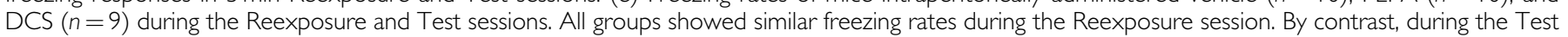

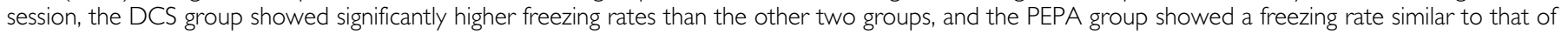

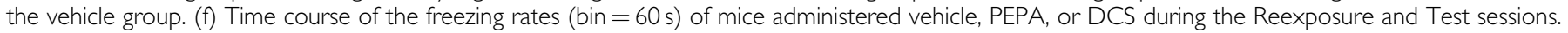

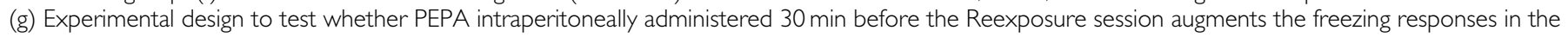

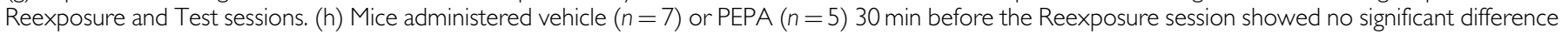

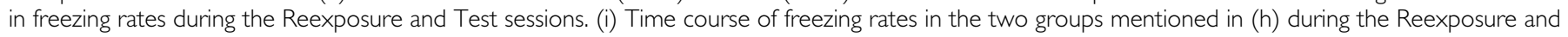

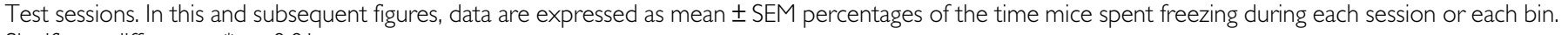
Significant difference; $* p<0.01$.

vehicle or PEPA 15 min before the Reexposure session, and the DCS group was similarly injected with DCS $30 \mathrm{~min}$ before the Reexposure session. In the experiment shown in Figure 1g-i, vehicle and PEPA groups were intraperitoneally administered vehicle and PEPA $30 \mathrm{~min}$ before the Reexposure session. On day 3 (Test), mice were reexposed to the conditioning chamber for $3 \mathrm{~min}$ to retrieve fear memory. In this and subsequent experiments, the duration of time mice spent frozen in the conditioning chamber was measured during playback of the VHS videotape. Complete immobilization of the mouse, except for respiration, was regarded as a freezing response (Blanchard and Blanchard, 1972). The freezing response was scored as the time mouse spent in freezing during a $180 \mathrm{~s}$ total session or a $60 \mathrm{~s}$ bin, and the amount of freezing response was expressed as the percent freezing rate in the 180 or $60 \mathrm{~s}$ time window.

Experiment 2: effect of PROP on the augmented fear responses induced by DCS. On day 1 (Conditioning), mice were conditioned using the methods described in Experiment 1 . On day 2 (Reexposure), before the Reexposure session, mice in one group were intraperitoneally administered vehicle $(n=11)$, and mice in other two groups were similarly administered DCS $(n=19)$. These mice were reexposed to the conditioning chamber for $3 \mathrm{~min}$. Immedi- ately after the Reexposure session, mice administered vehicle were intraperitoneally injected with saline; 10 out of 19 mice administered DCS were similarly injected with saline, and the other 9 were similarly injected with PROP. On day 3 (Test), these mice were reexposed to the conditioning chamber for $3 \mathrm{~min}$.

Experiment 3: microinjection of PEPA and DCS into the amygdala and its effect on reconsolidation. Microinjection into the amygdala was carried out as we reported previously (Zushida et al, 2007). Briefly, each mouse was anesthetized with pentobarbital $(50 \mathrm{mg} / \mathrm{kg}$, intraperitoneal) and affixed to the brain stereotaxic apparatus (Narishige Instruments, Tokyo, Japan). Stainless-steel guide cannulas (Eicom, Kyoto, Japan) were bilaterally inserted into the brain so that its tip was positioned into the amygdala (anteroposterior, $-1.22 \mathrm{~mm}$ from the bregma; lateral, $\pm 3.10 \mathrm{~mm}$; ventral, $-4.50 \mathrm{~mm}$ ) (Paxinos and Franklin, 2001). The cannulas were held in place with acrylic dental cement. A dummy cannula was inserted into the guide cannula to prevent clogging. These mice were fear conditioned 3 or 4 days after the surgery, and the conditioned mice were administered $(0.2 \mu \mathrm{l}$ per side, $0.05 \mu \mathrm{l} / \mathrm{min}) \mathrm{PEPA}$, DCS, or vehicle $15 \mathrm{~min}$ before the Reexposure session, which was carried out $24 \mathrm{~h}$ after the Conditioning session. The Test session was carried 

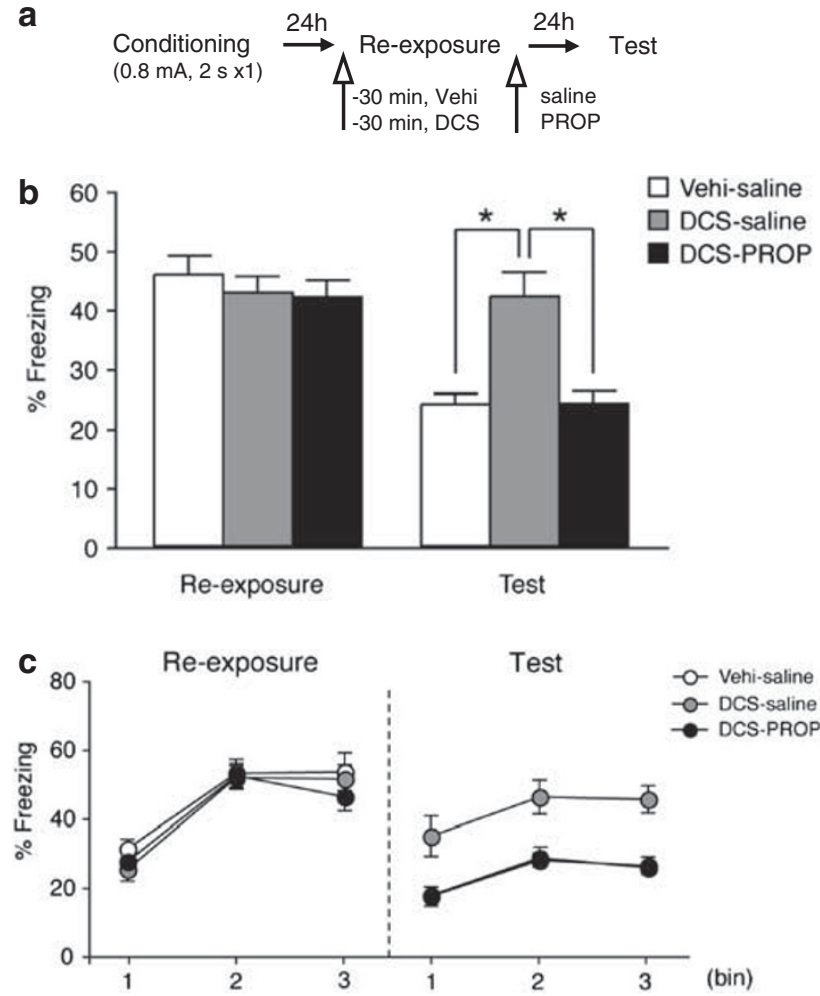

Figure 2 The D-cycloserine (DCS)-induced enhancement of the fear response was blocked by the $\beta$-adrenoceptor antagonist PROP. (a) Experimental design of this experiment. Vehicle (Vehi) or DCS was administered to conditioned mice 30 min before the Reexposure session, and saline or PROP was administered to the mice immediately after the Reexposure session. (b) Freezing rates of mice intraperitoneally administered vehicle and subsequent saline $(n=\mid I)$, DCS and subsequent saline $(n=10)$, or DCS and subsequent PROP $(n=9)$, in the Reexposure and Test sessions. All groups showed similar freezing rates during the Reexposure session. By contrast, during the Test session, the DCS-saline group showed significantly higher freezing rates than the vehicle-saline and DCS-PROP groups, and there was no significant difference in the freezing rates between vehicle-saline and DCS-PROP groups. (c) Time course of freezing rates (bin $=60 \mathrm{~s}$ ) during the Reexposure and Test sessions. Significant difference; $* 00.01$.

out $24 \mathrm{~h}$ after the Reexposure session. After the behavioral test, mice were anesthetized with halothane and microinjected with $0.2 \mu \mathrm{l}$ per side of fast green. The brains were isolated and sectioned $(200 \mu \mathrm{m})$ using a Vibratome 3000 (Vibratome, St Louis, MO). Cannula location was assessed under a light microscope.

Experiment 4: effects of PEPA and DCS on extinction and reinstatement of contextual fear. On day 1 (Conditioning), contextual fear conditioning was conducted as previously described (Zushida et al, 2007) with slight modifications. Mice were placed in the conditioning chamber and received a series of eight footshocks $(0.8 \mathrm{~mA}, 1 \mathrm{~s}$ duration) at an average of $30 \mathrm{~s}$ intervals. The first footshock was given $59 \mathrm{~s}$ after placement in the chamber. Mice were removed from the chamber $30 \mathrm{~s}$ after the end of the footshock and returned to their home cages. Freezing rates during this $30 \mathrm{~s}$ just after the footshocks were measured in some mice and are shown as post-shock freezing rates in Figure $4 \mathrm{~b}$. On days 2 and 3 (Extinction Trainings 1 and 2), mice were reexposed to the conditioning chamber without footshock for $6 \mathrm{~min}$ for extinction training. Mice in two groups were intraperitoneally injected with PEPA or vehicle $15 \mathrm{~min}$ before the first extinction training, and mice in another group were similarly injected with DCS $30 \mathrm{~min}$ before the first extinction training. On day 5 (Pre-reminder), mice were returned to the conditioning chamber for $3 \mathrm{~min}$ and tested for extinction retention. Then, a single footshock $(0.8 \mathrm{~mA}, 1 \mathrm{~s}$ duration) was delivered as a reminder shock. On day 6 (Test), mice were tested in the conditioning chamber for $6 \mathrm{~min}$. The scoring and quantification of freezing responses were carried out similarly to Experiment 1, except that, in the majority of the cases, the total session time was $360 \mathrm{~s}$.

Experiment 5: effects of PEPA and DCS on reextinction of contextual fear. On day 1 (Conditioning), each mouse was placed in the conditioning chamber and received footshocks as in Experiment 4. Mice were removed from the chamber $30 \mathrm{~s}$ after the end of the footshock and returned to their home cages. On day 2 (Extinction Training 1), mice were reexposed to the conditioning chamber without footshock for $6 \mathrm{~min}$ for extinction training. On day 4 (Test 1), mice were returned to the conditioning chamber for $6 \mathrm{~min}$ for testing of the extent of extinction. On day 5 (Conditioning 2 ), the same electrical footshocks used on day 1 were applied to the mice. On day 6 (Extinction Training 2), reextinction training was performed as on day 2 . At 15 or $30 \mathrm{~min}$ before the reextinction training, mice were intraperitoneally injected with PEPA or DCS; vehicle was similarly administered $15 \mathrm{~min}$ before the reextinction training. On day 8 (Test 2), mice were tested for extinction for $6 \mathrm{~min}$.

\section{Statistical Analysis}

All data are shown as means \pm SEM. One- or two-way analysis of variance (ANOVA) was used for comparisons of more than two groups. The factors in two-way ANOVAs were administration timing (15 and $30 \mathrm{~min}$ ) and session (Reexposure and Test) in the case of Figure 1b; drug (vehicle, PEPA, and DCS) and session (Reexposure and Test) in the case of Figures 1e, h, and 3b; and drug (vehiclesaline, DCS-saline and DCS-PROP) and session (Reexposure and Test) in the case of Figure 2b. Similarly, in the case of Figures $4 \mathrm{c}$ and $5 \mathrm{~b}$, the factors were drug (vehicle, PEPA, and DCS) and session (Extinction Trainings 1 and 2, Prereminder, and Test in Figure 4c; Extinction Trainings 1 and 2, Tests 1 and 2 in Figure $5 \mathrm{~b}$ ). In the case of Figure $4 \mathrm{~b}$, the factor in one-way ANOVA was drug groups. If the result of ANOVA was significant, Tukey's post hoc comparison test was used to analyze the freezing responses. $p<0.05$ was considered statistically significant.

\section{RESULTS}

\section{Intraperitoneally Administered PEPA does not Facilitate Fear Memory Reconsolidation}

To estimate reconsolidation, short 3-min reexposures were conducted in each conditioned mouse using the time schedule described in Experiment 1 in the Materials and Methods section, and the freezing rate (\%) during the $3 \mathrm{~min}$ period was measured. Because we used different 

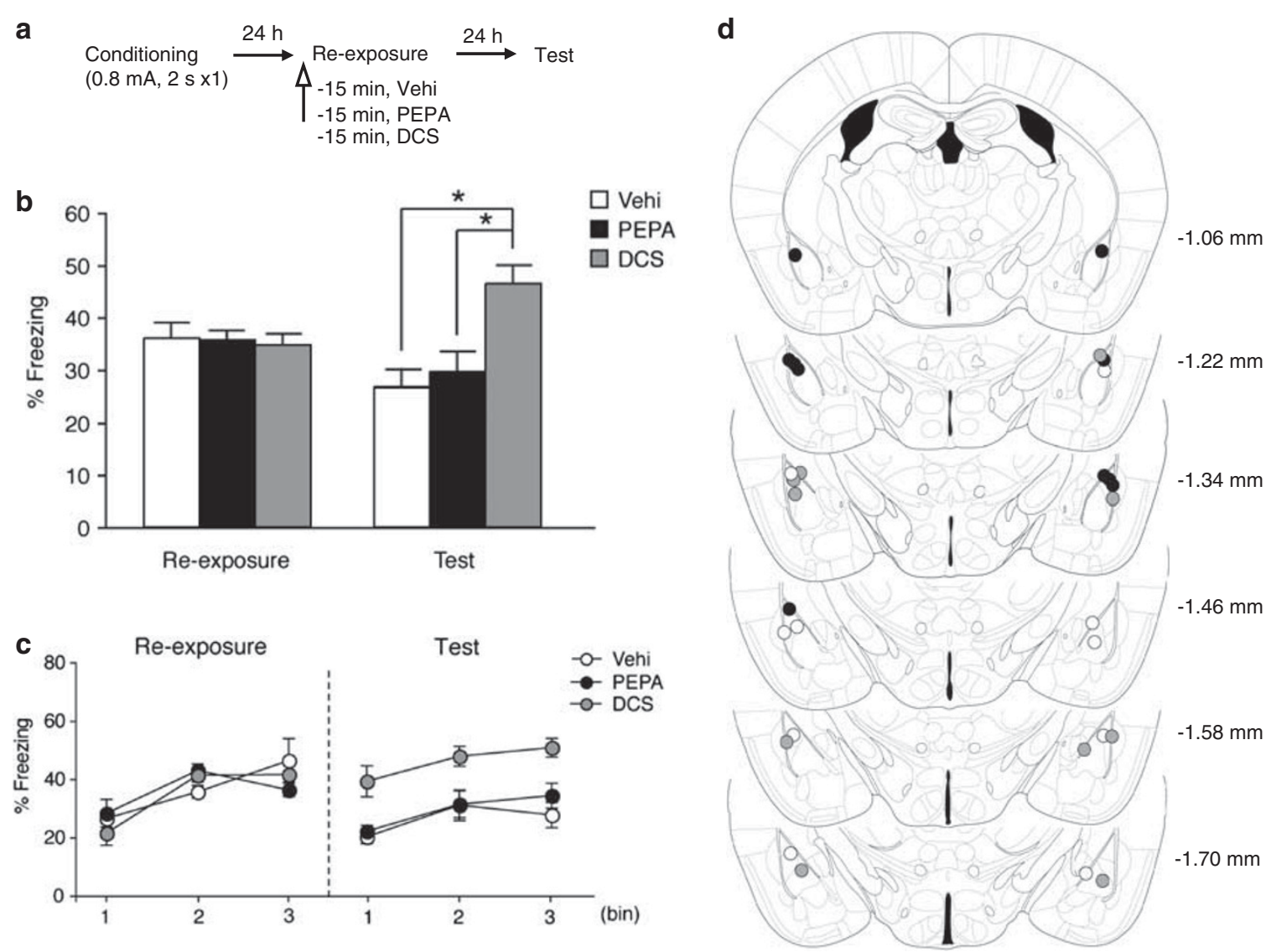

Figure 3 Intra-amygdala administration of 4-[2-(phenylsulfonylamino)ethylthio]-2,6-difluoro-phenoxyacetamide (PEPA) did not facilitate fear memory reconsolidation. (a) Experimental design of this experiment. (b) Freezing rates of the mice bilaterally administered vehicle $($ Vehi; $n=5)$, PEPA ( $n=5$ ), or D-cycloserine (DCS) $(n=5)$ into the amygdala ( 15 min before the Reexposure session), during the Reexposure and Test sessions. The freezing rates of all groups did not differ during the Reexposure session. During the Test session, the PEPA group showed no significant difference in freezing rate compared with the vehicle group. By contrast, the DCS group showed a significantly higher freezing rate than the vehicle and PEPA groups. (c) Time course of freezing rate (bin = $60 \mathrm{~s}$ ) during the Reexposure and Test sessions. (d) The position of the tip of the cannula inserted into the amygdala for injection. The numbers indicate the posterior coordinate from the bregma. Open circles indicate the vehicle group, closed circles the PEPA group, and shaded circles the DCS group. Significant difference; $* p<0.01$.

administration timings for PEPA $(-15 \mathrm{~min})$ and DCS $(-30 \mathrm{~min})$, which were based on previous studies on the action of PEPA on extinction learning (Zushida et al, 2007) and the action of DCS on extinction learning and reconsolidation (Walker et al, 2002; Lee et al, 2006), we examined whether administration of vehicle with different timings caused any differences in mouse freezing responses in the Reexposure session and subsequent Test session (Figure 1a). There was no significant difference $(\mathrm{F}<1$, two-way ANOVA, administration timing $\times$ session) in the freezing rates between the two groups administered vehicle 15 or $30 \mathrm{~min}$ before the Reexposure session (see Figure $1 \mathrm{~b}$ for the mean freezing rate in the $3 \mathrm{~min}$ session and Figure 1c for the time course, bin $=60 \mathrm{~s}$, in the $3 \mathrm{~min}$ session). Based on this result, vehicle-administration $15 \mathrm{~min}$ before the Reexposure session was used as the vehicle control for both PEPA and DCS administrations (Figure 1d). Figure 1e shows the freezing rates of mice during the Reexposure and Test sessions. The freezing rates during the Reexposure session were not significantly different among vehicle $(n=10)$, PEPA $(n=10)$, and DCS $(n=9)$ groups, suggesting that initial retrieval of fear memory is not altered by either
PEPA or DCS in this procedure (Figure 1e). However, twoway ANOVA (drug $\times$ session) revealed a significant effect of drug $\left(\mathrm{F}_{(2,52)}=3.25, p<0.05\right)$, a significant effect of session $\left(\mathrm{F}_{(1,52)}=12.79, p<0.01\right)$, and a significant drug $\times$ session interaction $\left(\mathrm{F}_{(2,52)}=10.46, p<0.01\right)$. In the Test session, a post hoc comparison indicated that the freezing rate in the DCS group was significantly higher than those in the other two groups $(p<0.01$ for DCS $v s$ vehicle, $p<0.01$ for DCS $v s$ PEPA), and the freezing rate in the PEPA group was not significantly different $(p>0.05)$ from that in the vehicle group (Figure 1e). Post hoc comparison also indicated that the vehicle and PEPA groups showed significantly lower freezing rates in the Test session than in the Reexposure session ( $p<0.05$ for vehicle, $p<0.01$ for PEPA), and that the DCS group showed similar freezing rates in the Reexposure and Test sessions $(p>0.05)$. Within-session analysis also revealed that the freezing rates in all groups were almost the same during the Reexposure session (bin $=60 \mathrm{~s}$ ), but in the Test session, the DCS group showed a persistently higher freezing rate than the vehicle and PEPA groups (Figure 1f).

Although these results suggest that PEPA administered $15 \mathrm{~min}$ before the Reexposure session does not increase 

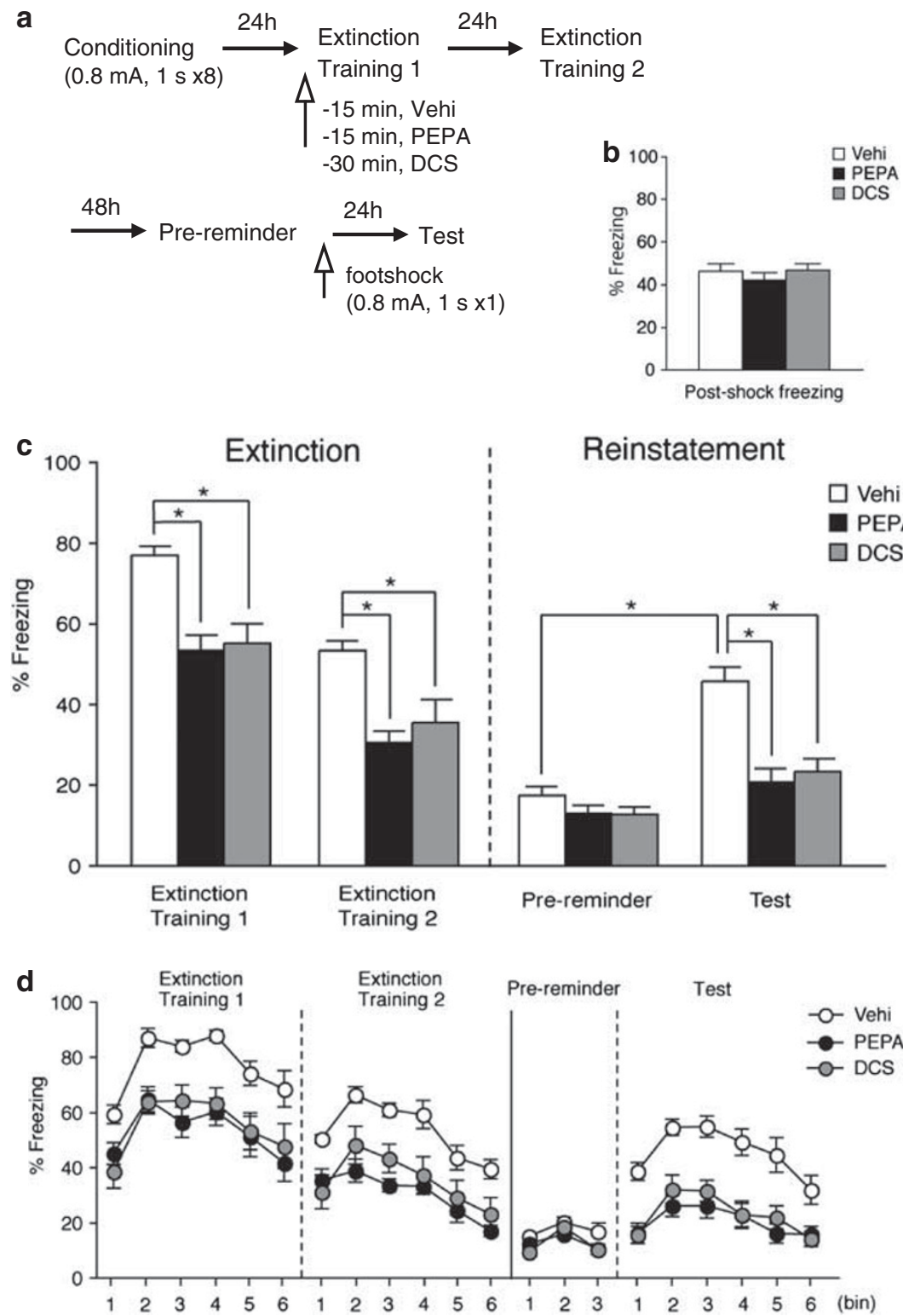

Figure 4 Direct comparison of the actions of 4-[2-(phenylsulfonylamino)ethylthio]-2,6-difluoro-phenoxyacetamide (PEPA) and D-cycloserine (DCS) on extinction and reinstatement of contextually conditioned fear. (a) Experimental design of this experiment. Vehicle (Vehi) and PEPA were administered I 5 min before the Extinction Training I session, and DCS was administered 30 min before the Extinction Training I session. (b) Freezing rates of some mice used in (c) during the $30 \mathrm{~s}$ immediately after the footshocks $(0.8 \mathrm{~mA}, \mathrm{Is}, 8 \times)$. These mice were randomly assigned to administration of vehicle, PEPA, and DCS ( $n=6$ each). (c, Extinction) Freezing rates of mice in the two Extinction Training sessions (vehicle, $n=11$; PEPA, $n=10$; or DCS, $n=10$ ). Both PEPA and DCS administered, respectively, I5 and 30 min before Extinction Training I, facilitated fear extinction during Extinction Training I and Extinction Training 2 with similar efficacy. (c, Reinstatement) The freezing rates of mice in the Pre-reminder session and Test session. At $48 \mathrm{~h}$ after Training 2 , the mice used in 'Extinction' were reexposed to the context for 3 min (Pre-reminder session), and a footshock weaker than that used in the original conditioning (reminder shock; $0.8 \mathrm{~mA},|\mathrm{~s}$,$| \times ) was applied just after the Pre-reminder session. The reminder shock induced significant augmentation of the freezing rate in the$ vehicle group, but not in the PEPA and DCS groups. (c) Time course of freezing rates (bin $=60 \mathrm{~s}$ ) during the two Extinction Training, Pre-reminder, and Test sessions. Significant difference; ${ }^{*} p<0.01$.

freezing rate in a subsequent test, in contrast to the increment induced by DCS, there remained a possibility that the administration timing of $15 \mathrm{~min}$ before the Reexposure session was too short for PEPA to act on reconsolidation (although PEPA was shown to facilitate extinction learning in mice with this administration timing; Zushida et al, 2007). To check this possibility, we carried out a similar experiment, but with administration of PEPA
$30 \mathrm{~min}$ before the Reexposure session (Figure 1g). No significant difference in freezing rate was observed between the two groups administered PEPA $(n=5)$ and vehicle $(n=7) 30$ min before the Reexposure session (see Figure $1 \mathrm{~h}$ for the mean freezing rate in the $3 \mathrm{~min}$ session and Figure 1i for the time course, bin $=60 \mathrm{~s}$, in the 3 min session). Twoway ANOVA (drug $\times$ session) revealed no significant effect of drug $(\mathrm{F}<1)$, no significant effect of session $\left(\mathrm{F}_{(1,20)}=3.48\right.$, 
a
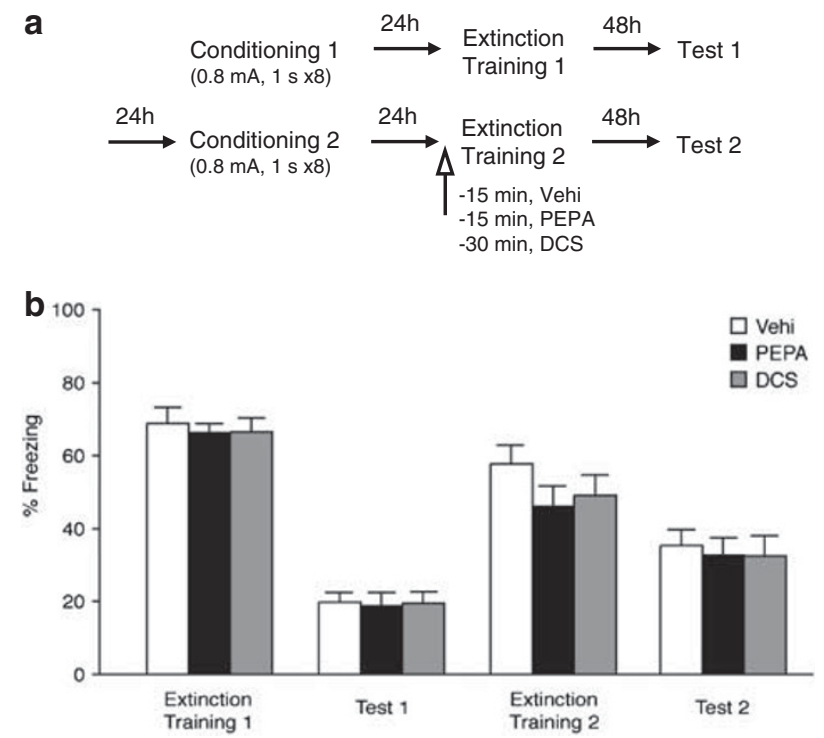

Figure 5 The actions of 4-[2-(phenylsulfonylamino)ethylthio]-2,6difluoro-phenoxyacetamide (PEPA) and D-cycloserine (DCS) on reextinction of contextually conditioned fear. (a) Experimental design of this experiment. The footshock of the same condition $(0.8 \mathrm{~mA}, 1 \mathrm{~s}, 8 \times)$ was applied to mice in conditioning sessions I and 2. Vehicle (Vehi, $n=9)$ and PEPA $(n=10)$ were intraperitoneally administered 15 min before Extinction Training 2, and DCS $(n=10)$ was similarly administered 30 min before Extinction Training 2. (b) Freezing rates of the mice during Extinction Training $\mid$ and Test I sessions for extinction of the first conditioning, and during Extinction Training 2 and Test 2 sessions for extinction of the second conditioning. Note that compared with vehicle neither PEPA nor DCS induced a reduction in freezing rates in the Test 2 session.

$p>0.05)$, and no significant drug $\times$ session interaction $(\mathrm{F}<1)$. From this result, we concluded that PEPA administered up to $30 \mathrm{~min}$ before the Reexposure session, unlike DCS, did not increase freezing rates in the subsequent Test session.

The higher freezing rate in the DCS group during the Test session was thought to result from facilitation of reconsolidation on reexposure. To confirm this, we administered PROP $(10 \mathrm{mg} / \mathrm{kg})$ to the DCS group immediately after the Reexposure session (Figure 2a). PROP administered immediately after reexposure inhibits fear memory reconsolidation, but not consolidation (Debiec and LeDoux, 2004). Figure $2 \mathrm{~b}$ shows the freezing rates (\%) of mice administered vehicle and subsequent saline, DCS and subsequent saline, and DCS and subsequent PROP, during 3 min Reexposure and Test sessions. The freezing rates during the Reexposure session were not statistically different among vehicle-saline $(n=11)$, DCS-saline $(n=10)$, and DCS-PROP $(n=9)$ groups (Figure 2b). Two-way ANOVA (drug $\times$ session) revealed a significant effect of drug $\left(\mathrm{F}_{(2,54)}=5.89\right.$, $p<0.01)$, a significant effect of session $\left(\mathrm{F}_{(1,54)}=32.13\right.$, $p<0.01)$, and a significant drug $\times$ session interaction $\left(\mathrm{F}_{(2,54)}=8.11, p<0.01\right)$. In the Test session, a post hoc comparison indicated that the DCS-saline group exhibited a higher freezing rate than the vehicle-saline and DCS-PROP groups ( $p<0.01$ for DCS-saline $v s$ vehicle-saline, $p<0.01$ for DCS-saline $v s$ DCS-PROP groups). On the other hand, there was no significant difference $(p>0.05)$ in freezing rates between the DCS-PROP and the vehicle-saline groups (Figure 2b). A post hoc test also indicated that the freezing rates of the vehicle-saline and DCS-PROP groups were significantly lower in the Test session than in the Reexposure session $(p<0.01$ for vehicle-saline, $p<0.01$ for DCS-PROP). Within-session analysis (bin $=60 \mathrm{~s}$ ) suggested a lower freezing rate in the DCS-PROP group than in the DCS-saline group in the Test session (Figure 2c). These results suggest that the augmented fear response in the DCS group was the result of facilitation of fear memory reconsolidation, and that PEPA, unlike DCS, did not facilitate the reconsolidation of fear memory.

\section{Intra-Amygdala Administration of PEPA does not Facilitate Fear Memory Reconsolidation}

To obtain more direct evidence for the hypothesis that PEPA does not act on reconsolidation, PEPA was bilaterally injected into the amygdalae of mice and behavioral experiments similar to those whose results are shown in Figure 1 were performed. Vehicle, PEPA, or DCS ( $n=5$ for each) was bilaterally injected into the amygdala $15 \mathrm{~min}$ before the Reexposure session through cannulae already inserted into the amygdala (Figure 3a). This administration timing was determined by a previous report on DCS (Walker et al, 2002). The freezing rates of the mice in the 3 min Reexposure and Test are shown in Figure 3b. During the Reexposure session, there were no significant differences in freezing rates among all three groups (Figure $3 b$ ). Two-way ANOVA (drug $\times$ session) revealed a significant effect of drug $\left(\mathrm{F}_{(2,24)}=6.01, p<0.01\right)$ and a significant drug $\times$ session interaction $\left(\mathrm{F}_{(2,24)}=7.45, p<0.01\right)$, but no significant effect of session $(\mathrm{F}<1)$. A post hoc comparison of results from the Test session indicated that the freezing rates of mice injected with PEPA did not differ from those of mice injected with vehicle alone $(p>0.05$ for PEPA $v s$ vehicle). By contrast, the freezing rates of mice injected with DCS in the Test session were significantly higher than those of mice injected with vehicle alone or PEPA $(p<0.01$ for DCS $v s$ vehicle, $p<0.01$ for DCS $v s$ PEPA) (Figure $3 \mathrm{~b}$ ). Within-session analysis also revealed that the freezing rates in all groups were almost the same during the Reexposure session (bin $=60 \mathrm{~s}$ ), but in the Test session, the DCS group showed persistently higher freezing rates than the vehicle and PEPA groups (Figure 3c). Figure $3 \mathrm{~d}$ shows the position of the tip of the cannula inserted into the amygdala for injection. These results suggest that PEPA, unlike DCS, has no effect on reconsolidation when it was directly administered into the amygdala.

\section{Comparison of the Actions of PEPA and DCS on Extinction of Contextual Conditioned Fear}

Given the possibility that PEPA selectively affects extinction and not reconsolidation, we directly compared the efficacies of PEPA and DCS (both at $30 \mathrm{mg} / \mathrm{kg}$ ) at facilitating extinction of contextual fear memory. Although these two compounds are known to facilitate extinction, they have never been compared in parallel in the same experiment. For this purpose, PEPA and DCS were intraperitoneally administered into conditioned mice 15 and $30 \mathrm{~min}$ before the first extinction training (Extinction Training 1, Figure 4a), respectively, and the freezing rates of mice were measured during two long $(6 \mathrm{~min})$ reexposures (Extinction 
Trainings 1 and 2 for extinction, Figure 4a). Similarly to the reconsolidation experiments, we examined the influence of vehicle administration with different timings (15 and $30 \mathrm{~min}$ before Training 1) on the freezing rates of mice, and found that there was no significant difference in freezing rate between the two groups (Supplementary Figure S1). Therefore, vehicle administration $15 \mathrm{~min}$ before Extinction Training 1 was used as the control (Figure 4a).

We also analyzed the freezing rates of mice during the $30 \mathrm{~s}$ immediately after the footshocks (Figure $4 \mathrm{~b}$ ). This analysis was carried out in some of the mice $(n=6$ for each of vehicle, PEPA, and DCS) used in the experiment, illustrated in Figure 4c, to check whether mice in three groups randomly assigned to administration of vehicle, PEPA, or DCS show similar levels of post-shock freezing (a very short-term memory assessment of contextual fear acquisition). As shown in Figure $4 \mathrm{~b}$, there was no significant difference in the mean freezing rates among three groups $(\mathrm{F}<1$, one-way ANOVA).

Figure 4c (Extinction) shows the freezing rates of mice administered vehicle $(n=11)$, PEPA $(n=10)$, or DCS $(n=10)$ during two 6 min extinction trainings. A two-way ANOVA (drug $\times$ session) of extinction data from Extinction Trainings 1 and 2 indicated significant effects of drug $\left(\mathrm{F}_{(2,56)}=25.26, \quad p<0.01\right)$ and session $\left(\mathrm{F}_{(1,56)}=56.24\right.$, $p<0.01)$. A post hoc comparison revealed that, in both Extinction Trainings 1 and 2, the PEPA and DCS groups exhibited freezing rates significantly lower than that of the vehicle group $(p<0.01$ for PEPA $v s$ vehicle, $p<0.01$ for DCS $v s$ vehicle). On the other hand, there was no significant difference in freezing rates between the PEPA and DCS groups, in Extinction Training 1 or $2(p>0.05)$. A post hoc test also indicated that the freezing rate in each group was significantly lower in Extinction Training 2 than in Extinction Training $1 \quad(p<0.01$ for vehicle, PEPA, and DCS, respectively). Within-session analysis (bin $=60 \mathrm{~s}$ ) revealed that the freezing rates in the PEPA and DCS groups were lower than the rate in the vehicle group throughout Extinction Trainings 1 and 2 (Figure 4c). These results suggest that PEPA and DCS, at the doses used, facilitate extinction with similar efficacies in our experimental system.

\section{PEPA and DCS Suppress the Reinstatement of Contextual Conditioned Fear}

The mice used above were subsequently used in a reinstatement test $(n=11,10$, and 10 for vehicle, PEPA, and DCS, respectively). Reinstatement has been considered to be a model for patients who reexhibit a fear response when they encounter a weak aversive stimulus (Ledgerwood et al, 2004). Because DCS is known to suppress the emergence of the reinstatement of conditioned fear (Ledgerwood et al, 2004), it is likely that examining the action of PEPA on reinstatement would be informative for the comparison of PEPA and DCS as extinction-facilitating drugs. At $48 \mathrm{~h}$ after Extinction Training 2, the mice were again reexposed to the context for $3 \mathrm{~min}$ (Pre-reminder session, Figure 4a). At the end of the Pre-reminder session, a weak electrical footshock (once, at the same amplitude used in the original conditioning) was applied as a 'reminder'; $24 \mathrm{~h}$ later, mice were reexposed to the context again to estimate reinstatement (Test session). The Prereminder session was designed to estimate the freezing rates of mice just before the reminder shock. Figure $4 \mathrm{~b}$ (Reinstatement) shows the freezing rates of the mice before and after the reminder shock. A two-way ANOVA (drug $\times$ session) of the results from the Pre-reminder session and the Test session revealed a significant effect of drug $\left(\mathrm{F}_{(2,56)}=22.16, p<0.01\right)$, a significant effect of session $\left(\mathrm{F}_{(1,56)}=57.99, p<0.01\right)$, and a significant drug $\times$ session interaction $\left(\mathrm{F}_{(2,56)}=10.35, p<0.01\right)$. A post hoc comparison revealed no significant differences in freezing rates among the three groups in the 'Pre-reminder' session $(p>0.05$; Figure $4 c$ ). In the Test session, the freezing rates of the mice in the vehicle group were significantly higher than those in the other two groups $(p<0.01$ for both vehicle $v s$ PEPA and vehicle vs DCS, Figure 4c). In addition, post hoc analysis suggested that the freezing rate of the vehicle group was significantly higher in the Test session than in the Prereminder session $(p<0.01)$, but in the case of PEPA and DCS groups, there was no significant difference in freezing rate between the Pre-reminder session and the Test session $(p>0.05)$. Within-session analysis $(\mathrm{bin}=60 \mathrm{~s})$ revealed that the freezing rates in the PEPA and DCS groups were lower than the rates in the vehicle group throughout the Test session (Figure 4c). These results suggest that PEPA and DCS suppressed the emergence of reinstatement in our experimental system.

\section{PEPA and DCS do not Facilitate Reextinction of Contextual Conditioned Fear}

It has been reported that DCS does not facilitate extinction a second time when the second extinction training is conducted using the same CS (light) (Langton and Richardson, 2008). Therefore, it is likely that examining the action of PEPA on reextinction would be informative for the comparison of PEPA and DCS as extinction-facilitating drugs. Figure 5 shows the effects of PEPA and DCS on reextinction (time schedule of experiments in Figure 5a). The mean freezing rates in the three groups of mice, which were randomly divided, were almost identical during the Extinction Training 1 and Test 1 sessions (Figure 5b). At $24 \mathrm{~h}$ after the second conditioning, the mice in the three groups were intraperitoneally administered vehicle $(n=9)$, PEPA $(n=10)$, or DCS $(n=10)$; they were then reexposed to the context in Extinction Training 2 (Figure $5 \mathrm{~b}$ ) and the subsequent Test 2. Two-way ANOVA (drug $\times$ session) revealed a significant effect of session $\left(\mathrm{F}_{(1,52)}=17.57\right.$, $p<0.01)$, but no significant effect of drug $\left(\mathrm{F}_{(2,52)}=1.07\right.$, $p>0.05)$ and no significant drug $\times$ session interaction $(\mathrm{F}<1)$. A post hoc comparison indicated that the freezing rates during Training 2 and test sessions were not statistically different among all groups; that is, the PEPA and DCS groups exhibited similar freezing rates to the vehicle group $(p>0.05$ for both PEPA $v s$ vehicle and DCS $v s$ vehicle). A post hoc test also indicated that the freezing rates of the mice in the vehicle group were significantly reduced in the Test 2 session compared with those in the Extinction Training 2 session $(p<0.05)$. The PEPA and DCS groups exhibited freezing rates that were not statistically different between the Extinction Training 2 and Test 2 sessions ( $p>0.05$ for both PEPA and DCS), unlike the case when 
drugs were administered before initial extinction training, as shown in Figure 4c. These results suggest that PEPA and DCS administered just before the second extinction training did not facilitate reextinction of contextual conditioned fear. The results of within-session analysis are shown in the Supplementary information, which is available at the Neuropsychopharmacology website (Supplementary Figure S2).

\section{DISCUSSION}

The main finding in the present study is that intraperitoneal administration of the AMPA potentiator PEPA facilitates extinction of contextual fear without any detectable influence on reconsolidation. Although reconsolidation and extinction are thought to be distinct processes, a compound that selectively acts on one of these processes following systemic administration has not been reported. The results of the present study demonstrate the existence of such a compound, indicating that extinction and reconsolidation can be pharmacologically discriminated.

As we reported previously, PEPA facilitates synaptic responses in $\mathrm{mPFC}$ layer $\mathrm{V}$ pyramidal neurons much more potently than those in BLA principal neurons and hippocampus CA1 pyramidal neurons (Zushida et al, 2007). In particular, PEPA induces input-dependent population firing only in the mPFC (Zushida et al, 2007). The facilitating action of PEPA on extinction can be explained by activation of the mPFC, which is one of the crucial factors in consolidation and retrieval of extinction (Milad and Quirk, 2002; Milad et al, 2004). A candidate molecular basis for the more potent electrophysiological activity of PEPA in the MPFC than in the BLA and CA1 is that the relative expression level of GluR3-flop mRNA, an AMPA receptor variant most vigorously potentiated by PEPA (Sekiguchi et al, 1997), is higher in the mPFC than in the BLA and CA1 (Zushida et al, 2007). The importance of the BLA in the reconsolidation of fear memory has been shown (Nader et al, 2000; Duvarci and Nader, 2004; Baldi et al, 2008), as mentioned in the Introduction, but participation of the mPFC has not been. Therefore, one explanation for the absence of an action of PEPA on reconsolidation in the present study is that PEPA is not so active in strengthening the BLA neuronal circuits necessary for reconsolidation. This inactiveness may be explained by a lower expression level of GluR3-flop in the BLA compared with the mPFC. Our present data showing that even intra-amygdala injection of PEPA does not enhance reconsolidation supports this explanation.

In the present study, we used unconditioned stimuli (US; footshock) with different strengths between the short reexposure experiments for reconsolidation and long reexposure experiments for extinction. This was performed to induce either reconsolidation or extinction predominantly. However, it is thought that there is a condition in which the US is able to effectively induce both reconsolidation and extinction with a temporal difference (for review, see Tronson and Taylor, 2007). Therefore, it should be noted that whether PEPA exerts selectivity in reconsolidation and extinction induced by the same US is still an open question.
Previous studies suggest that systemic administration of AM404, an inhibitor of cannabinoid breakdown and reuptake, enhances extinction of conditioned fear measured with fear-potentiated startle in rats without changing the expression of conditioned fear itself, and that systemic administration of the CB1 cannabinoid receptor agonist WIN55212-2 does not appear to affect this extinction (Chhatwal et al, 2005). Furthermore, when administered by intrahippocampal injection, the CB1 cannabinoid receptor agonist anandamide blocks reconsolidation and facilitates extinction of contextual fear measured with freezing response in rats (De Oliveira Alvares et al, 2008), and when administered by intra-amygdala injection, WIN55212-2 blocks reconsolidation of fear memory measured with fear-potentiated startle (Lin et al, 2006). Although not demonstrated yet, these reports imply the possibility that particular compounds that act on the cannabinoid system may selectively modulate either reconsolidation or extinction following their systemic administration. The identification of such selective compounds with an action point distinct from PEPA may be informative for the development of therapeutic drugs for anxiety disorders.

In a direct comparison of the effects of PEPA and DCS on simple extinction learning, these compounds, at the same dose $(30 \mathrm{mg} / \mathrm{kg})$, exerted similar efficacies to facilitate extinction of contextual fear. However, the present study does not address whether PEPA can facilitate extinction of fear memory associated with a CS other than context. DCS is reported to enhance extinction of conditioned fear memory formed by light or tone as a CS (Walker et al, 2002; Ledgerwood et al, 2003; Langton and Richardson, 2008). The dependency of the extinction-facilitating action of PEPA on variety of conditioned stimuli should be addressed for a better understanding of the action of PEPA on extinction.

Reinstatement of fear is thought to be one phenomenon indicating that extinction does not lead to an erasure of original conditioned fear memory, but to new inhibitory learning for the original fear memory (for a review, see Myers and Davis, 2002). According to this interpretation, PEPA suppresses reinstatement through a mechanism that increases the strength of inhibitory association learning. Another interpretation for suppressing reinstatement by PEPA may be that dissociation of the original CS-US association (unlearning) is enhanced as a secondary effect induced by the facilitation of inhibitory learning (extinction). Notably, the suppression of reinstatement has also been reported for AM404 (Chhatwal et al, 2005). Because PEPA, DCS, and AM404 all suppress reinstatement in spite of their distinct sites of action, AMPA receptors, NMDA receptors, and the cannabinoid system, respectively, suppressing reinstatement may be a common property of extinction-facilitating compounds, regardless of their site of action.

Reextinction describes the phenomenon in which the fear response, which is reestablished by a further conditioning session following the initial extinction session, is again attenuated by reexposure to the original CS. One plausible explanation for the absence of an action of PEPA on reextinction is as follows. Several studies suggest that the BLA is not required for reextinction (Laurent et al, 2008; Laurent and Westbrook, 2008; Kim and Richardson, 2008). 
Because PEPA activates the MPFC to facilitate the inhibition of original association learning that is formed in the BLA, in cases in which the BLA is not involved, the activation of the mPFC by PEPA would become meaningless. The absence of an action of PEPA on reextinction thus supports the view that reextinction uses neuronal circuits distinct from those used during initial extinction (Langton and Richardson, 2008).

In summary, in the present study, we identified the AMPA potentiator PEPA as a candidate extinction-facilitating compound with no effect on reconsolidation of contextual fear memory. PEPA, as well as DCS, suppressed reinstatement of fear memory, but did not influence reextinction. Facilitation of reconsolidation is the most undesired side effect of extinction-facilitating drugs when they are used to enhance exposure therapy. Drug-induced enhancement of exposure therapy for the treatment of anxiety disorders could be improved by the development of such extinction-specific therapeutic drugs.

\section{ACKNOWLEDGEMENTS}

This work was supported by a Grant-in-Aid for Scientific Research (KAKENHI, 19500288) and the Program for Promotion of Fundamental Studies in Health Sciences of the National Institute of Biomedical Innovation, and a grant from the Japan Science and Technology Agency (CREST).

\section{DISCLOSURE/CONFLICT OF INTEREST}

The author(s) declare that no financial support or compensation has been received from any individual or corporate entity over the past 3 years for research or professional service, and that there are no personal financial holdings that could be perceived as constituting a potential conflict of interest.

\section{REFERENCES}

Baldi E, Mariottini C, Bucherelli C (2008). Differential roles of the basolateral amygdala and nucleus basalis magnocellularis during post-reactivation contextual fear conditioning reconsolidation in rats. Neurobiol Learn Mem 90: 604-609.

Blanchard RJ, Blanchard DC (1972). Innate and conditioned reactions to threat in rats with amygdaloid lesions. J Comp Physiol Psychol 81: 281-290.

Chhatwal JP, Davis M, Maguschak KA, Ressler KJ (2005). Enhancing cannabinoid neurotransmission augments the extinction of conditioned fear. Neuropsychopharmacology 30: 516-524.

Corcoran KA, Quirk GJ (2007). Recalling safety: cooperative functions of the ventromedial prefrontal cortex and the hippocampus in extinction. CNS Spectr 12: 200-206.

Davis M, Myers KM, Chhatwal J, Ressler KJ (2006). Pharmacological treatments that facilitate extinction of fear: relevance to psychotherapy. NeuroRX 3: 82-96.

Debiec J, LeDoux JE (2004). Disruption of reconsolidation but not consolidation of auditory fear conditioning by noradrenergic blockade in the amygdala. Neuroscience 129: 267-272.

De Oliveira Alvares L, Genro BP, Diehl F, Molina VA, Quillfeldt JA (2008). Opposite action of hippocampal CB1 receptors in memory reconsolidation and extinction. Neuroscience 154: $1648-1655$.
Diergaarde L, Schoffelmeer ANM, De Vries TJ (2008). Pharmacological manipulation of memory reconsolidation: towards a novel treatment of pathogenic memories. Eur J Pharmacol 585: 453-457.

Duvarci S, Nader K (2004). Characterization of fear memory reconsolidation. J Neurosci 24: 9269-9275.

Guastella AJ, Richardson R, Lovibond PF, Rapee RM, Gaston JE, Mitchell $\mathrm{P}$ et al (2008). A randomized controlled trial of D-cycloserine enhancement of exposure therapy for social anxiety disorder. Biol Psychiatry 63: 544-549.

Hofmann SG, Meuret AE, Smits JA, Simon NM, Pollack MH, Eisenmenger $\mathrm{K}$ et al (2006). Augmentation of exposure therapy with D-cycloserine for social anxiety disorder. Arch Gen Psychiatry 63: 298-304.

Kim JH, Richardson R (2008). The effect of temporary amygdala inactivation on extinction and reextinction of fear in the developing rat: unlearning as a potential mechanism for extinction early in development. $J$ Neurosci 28: 1282-1290.

Kushner MG, Kim SW, Donahue C, Thuras P, Adson D, Kotlyar M et al (2007). D-cycloserine augmented exposure therapy for obsessive-compulsive disorder. Biol Psychiatry 62: 835-838.

Langton JM, Richardson R (2008). D-cycloserine facilitates extinction the first time but not the second time: an examination of the role of NMDA across the course of repeated extinction sessions. Neuropsychopharmacology 33: 3096-3102.

Laurent V, Marchand AR, Westbrook RF (2008). The basolateral amygdala is necessary for learning but not relearning extinction of context conditioned fear. Learn Mem 15: 304-314.

Laurent V, Westbrook RF (2008). Distinct contributions of the basolateral amygdala and the medial prefrontal cortex to learning and relearning extinction of context conditioned fear. Learn Mem 15: 657-666.

Ledgerwood L, Richardson R, Cranney J (2003). Effects of D-cycloserine on extinction of conditioned freezing. Behav Neurosci 117: 341-349.

Ledgerwood L, Richardson R, Cranney J (2004). D-cycloserine and the facilitation of extinction of conditioned fear: consequences for reinstatement. Behav Neurosci 118: 505-513.

Lee JLC, Milton AL, Everitt BJ (2006). Reconsolidation and extinction of conditioned fear: inhibition and potentiation. J Neurosci 26: 10051-10056.

Lin HC, Mao SC, Gean PW (2006). Effects of intra-amygdala infusion of $\mathrm{CB} 1$ receptor agonists on the reconsolidation of fearpotentiated startle. Learn Mem 13: 316-321.

Milad MR, Quirk GJ (2002). Neurons in medial prefrontal cortex signal memory for fear extinction. Nature 420: 70-74.

Milad MR, Vidal-Gonzalez I, Quirk GJ (2004). Electrical stimulation of medial prefrontal cortex reduces conditioned fear in a temporally specific manner. Behav Neurosci 118: 389-394.

Mueller D, Porter JT, Quirk GJ (2008). Noradrenergic signaling in infralimbic cortex increases cell excitability and strengthens memory for fear extinction. J Neurosci 28: 369-375.

Myers KM, Davis M (2002). Behavioral and neural analysis of extinction. Neuron 36: 567-584.

Myers KM, Davis M (2007). Mechanisms of fear extinction. Mol Psychiatry 12: 120-150.

Nader K, Schafe GE, LeDoux JE (2000). Fear memories require protein synthesis in the amygdala for reconsolidation after retrieval. Nature 406: 722-726.

Norberg MM, Krystal JH, Tolin DF (2008). A meta-analysis of D-cycloserine and the facilitation of fear extinction and exposure therapy. Biol Psychiatry 63: 1118-1126.

Ouyang M, Thomas SA (2005). A requirement for memory retrieval during and after long-term extinction learning. Proc Natl Acad Sci USA 102: 9347-9352.

Paxinos G, Franklin KBJ (2001). The Mouse Brain in Stereotaxic Coordinates 2nd edn Academic Press: San Diego, CA, USA. 
Quirk GJ, Mueller D (2008). Neural mechanisms of extinction learning and retrieval. Neuropsychopharmacology 33: 56-72.

Ressler KJ, Rothbaum BO, Tannenbaum L, Anderson P, Graap K, Zimand E et al (2004). Cognitive enhancers as adjuncts to psychotherapy: use of D-cycloserine in phobic individuals to facilitate extinction of fear. Arch Gen Psychiatry 61: 1136-1144.

Sara SJ (2000). Retrieval and reconsolidation: toward a neurobiology of remembering. Learn Mem 7: 73-84.

Sekiguchi M, Fleck MW, Mayer ML, Takeo J, Chiba Y, Yamashita S et al (1997). A novel allosteric potentiator of AMPA receptors: 4-[2-(phenylsulfonylamino)ethylthio]-2,6-difluoro-phenoxyaceta mide. J Neurosci 17: 5760-5771.

Sotres-Bayon F, Bush DEA, LeDoux JE (2004). Emotional perseveration: an update on prefrontal-amygdala interactions in fear extinction. Learn Mem 11: 525-535.
Suzuki A, Josselyn SA, Frankland PW, Masushige S, Silva AJ, Kida S (2004). Memory reconsolidation and extinction have distinct temporal and biochemical signatures. J Neurosci 24: 4787-4795.

Tronson NC, Taylor JR (2007). Molecular mechanisms of memory reconsolidation. Nat Rev Neurosci 8: 262-275.

Vervliet B (2008). Learning and memory in conditioned fear extinction: effects of D-cycloserine. Acta psychologica (Amst) 127: 601-613.

Walker DL, Ressler KJ, Lu KT, Davis M (2002). Facilitation of conditioned fear extinction by systemic administration or intraamygdala infusions of $\mathrm{D}$-cycloserine as assessed with fearpotentiated startle in rats. J Neurosci 22: 2343-2351.

Zushida K, Sakurai M, Wada K, Sekiguchi M (2007). Facilitation of extinction learning for contextual fear memory by PEPA: a potentiator of AMPA receptors. J Neurosci 27: 158-166.

Supplementary Information accompanies the paper on the Neuropsychopharmacology website (http://www.nature.com/npp) 